TOPIC BRIEFS AND PERSPECTIVES

\title{
Clinical Data Management: A Review of Current Practice in Australia
}

\author{
Lauren Houston ${ }^{*},+\neq$ and Yasmine Probst ${ }^{*}, \dagger$
}

The practice of clinical data management (CDM) in Australia has seen and continues to experience tremendous growth. As such, this article reviews the current practice of CDM in Australia. The article addresses the history of the profession and provides insight into the difference between the sectors, the evolving role, ongoing requirements for training and education, and an overview of the regulations and how these impact the Australian CDM landscape. Current CDM practice in Australia differs considerably across industry, academic, and non-profit sectors though uniform regulatory requirements are provided nationwide. This has raised challenges for mostly academic, non-profit, and small-scale trials, which are more likely to lack access to resources, facilities, management, and funding. Australian clinical data managers are required to have formal skills related to data, technology, security, and project management, though they are also expected to operate at the highest levels of excellence across all areas of their diverse roles. It is only in recent years that CDM has evolved to a stronger focus on data quality. Regardless of these challenges, clinical data managers have played, and continue to play, a key role in Australian biomedical research. They have provided guidance on data collection, processing, and management procedures to ensure that studies achieve high quality outcomes. However, more research is needed to develop specific CDM training courses to help Australian clinical data managers meet a standard of knowledge, education, and experience to be officially recognised as a profession.

Keywords: Data management; Clinical trial; Biomedical research; Australia; Manage clinical research data

\section{Introduction}

The practice of clinical data management (CDM) in Australia is still evolving into an established discipline and career pathway. Australian clinical data managers come from a variety of educational qualifications and levels of professional experience, including backgrounds of computer science, life science, biostatistics, health informatics, and engineering. They require formal skills related to data, technology, security, and project management. Due to these hybrid requirements, clinical data managers are vital to many research institutes. Over the years, clinical data managers have had to keep up with the rapid growth in technology, the integration and handling of novel data streams, and the training of professionals with a diverse skillset. These requirements have expanded clinical

\footnotetext{
* School of Medicine, Faculty of Science, Medicine and Health, University of Wollongong, Northfields Ave, Wollongong, NSW 2522, AU

Illawarra Health and Medical Research Institute, University of Wollongong, Wollongong, NSW 2522, AU

₹ The George Institute for Global Health, University of New South Wales, Sydney, NSW 2050, AU

Corresponding author: Lauren Houston, PhD, BSc (Hons) (lah993@uowmail.edu.au)
}

data managers' responsibilities and expected expertise. The present article addresses an Australian perspective of the CDM profession. The article has been developed based on a history of the profession and provides insights into the differences between sectors, its evolving role, regulations in Australia, and their impact on the CDM landscape. Finally, this article provides an overview of the requirements for CDM training and education.

\section{History of the Profession in Australia}

Over the last two decades, the Australian CDM landscape has changed dramatically. At the heart of these changes has been the advancement of information technology (IT) systems and new data sources. The shift has led to a need to upskill Australian clinical data managers, which has seemingly progressed quicker within industry than for the academic, government, or non-profit sectors. The pharmaceutical industry has set the standard for CDM largely due to its access to resources, facilities, management, and funding. By comparison, academic clinical studies have generally been slower to adopt data management plans and specialist software due to limited time and resources. ${ }^{1-3}$ In 2018, a national survey found that Australian academic clinical trials were more likely to implement smaller, specialised, in-house systems 
(e.g., REDCap) ${ }^{2}$ rather than sophisticated CDM systems commonly utilised within the pharmaceutical industry (e.g., Oracle Clinical).

At present within the Australian clinical sector, all research institutes are provided with the same CDM advice. Research institutes have the responsibility to develop and implement data management policies. They also should provide facilities and processes for the safe and secure storage and management of their data. ${ }^{4}$ Australian regulatory agencies acknowledge the variability in size, maturity, experience, and organisational structure of clinical research institutes. However, the current advice for how to practice CDM is challenging for some due to the regulatory requirements endorsing the use of IT systems. In addition, there is a shift towards more complex protocols and newer data sources.

In Australia, it is known that large-scale clinical studies have tended to move towards electronic data capture (EDC) systems or have accumulated implementation expertise with IT systems more rapidly than smaller-scale clinical studies. ${ }^{5}$ For small studies, it is more likely to see a continued presence of traditional paper-based data capture methods as a perceived effective strategy. As the same requirements are necessary in Australia regardless of the study design or clinical focus, small-scale clinical studies need to consider the additional costs that operating an EDC system may incur for staff training, maintenance, support, hardware, and security requirements.

\section{How Work Varies across the Australian Industry, Academic, and Non-profit Sectors}

Within the Australian clinical trial landscape, there are two distinct yet interdependent sectors: industry-sponsored trials, such as commercial trials (e.g., medical technology, biotechnology, and pharmaceutical trials), and nonindustry-sponsored trials, such as investigator-initiated trials (e.g., academic, collaborative group, and non-profit trials). ${ }^{6}$ Typically, industry trials are conducted by separate entities, with sponsors having no direct involvement in the trial. Industry-led trials have strict requirements to uphold due to their funding and sponsorship agreements. On the other hand, academic studies are often smaller and more flexible to incremental change. ${ }^{5}$ Academic- and non-profit-led clinical studies are known to fluctuate in their funding availability and are often driven by external influences.

Standards and requirements of academic and non-profit trials are often internally driven by resource availability, with many researchers holding dual roles within their organisations. They usually rely largely on government (e.g., public) funding, philanthropic donations, and funding institutions (e.g., universities and hospitals). It is common that the sponsor of academic and non-profit trials is the person that developed the trial protocol. Therefore, the sponsor is responsible for the entire management of the trial, including the investment in technology and costeffective solutions to CDM.

Regardless of the sector, within CDM there has been miscommunication and a duplication of effort due to a tendency to work in silos as individuals meet the requirements of internal standards. Even between organisations, relative consistency is uncommon despite common regulatory systems. In recent years, however, a gradual influx of seeming consistency has begun to occur from an awareness of the need for standardised data frameworks.

To streamline approaches and to minimise the duplication of effort, the Clinical Trials Project Reference Group (CTPRG) was established in 2014. The CTPRG involves senior officials from commonwealth, state, and territory health departments, as well as the National Health and Medical Research Council (NHMRC), the Australian Government's primary health and medical research funding agency. ${ }^{7}$ While the common goal is to create processes to streamline systems nationally, some organisations are further ahead than others.

Currently, the industry sector is ahead of the academic and non-profit sectors. One of the factors contributing to this is the additional funding, which allows for the adoption of IT systems and access to staff training for these systems. The implementation of IT systems allows for real-time data collection and monitoring, improving the timeliness of the data. Therefore, industry-led trials are often faster-paced as IT systems allow for more rapid data analyses, leading to increased time to write and distribute research findings. This may only be part of the equation, however, as talented staff and streamlined systems emphasising cost containment may also play a role in keeping industry ahead of academic and nonprofit sectors. It is also worth considering the impact of globalisation on research; offshoring, mergers, and shifting priorities may also derail clinical research studies, as well as their results, conclusions, and recommendations. This is a potential limitation for Australia, as industry-led trials are now looking to move studies to emerging neighbouring regions, such as Asia. ${ }^{8}$ Such moves may result in reduced operational costs while still recruiting a large number of study participants in a timely manner.

\section{Role of Biomedical Research in Australia's Economic and Scientific Enterprise}

Australia has a proud history of scientific advancements arising from biomedical research. Australian scientists have played pivotal roles in the development of penicillin as an antibiotic as well as other medical breakthroughs, including the bionic ear, grey scale ultrasound imaging, spray-on skin, and the cervical cancer vaccine. ${ }^{9}$ While all of the listed biomedical research studies have collected, processed, and managed research data, there have been vastly different requirements for CDM between the past and present. In 1928, when penicillin was discovered, there was no published evidence relevant to CDM. It wasn't until 1990 that the first paper on data collection and processing methods for existing clinical research databases was available. ${ }^{10}$ Many years later, in 2019, the Australian government published its first guidance document for clinical researchers on the management of data and information in research. ${ }^{4}$ Today, however, there is a growing body of work in CDM that is appropriate to current practice. 
The Australian clinical trials sector is growing. Between 2010 and 2015 there was an increase in the amount of clinical trials by approximately $5 \%$ each year. ${ }^{6}$ This growth is faster than that in the US and the UK, as well as the average global rate. In 2015, the investment in Australian clinical trials accounted for roughly AU\$1.1 billion ( US\$824 million) in gross expenditure, with the majority (AU\$930 million, US\$697 million) of funding invested by international industry sponsors. These sponsors included medical device, biotechnology, and pharmaceutical companies. In total, the clinical trials sector supported at least 6,900 high-skilled jobs in 2015. In the next 10 years, it is expected that the Australian clinical trial sector will surpass AU\$2 billion ( US\$1.5 billion) of annual expenditure and create more than 6,000 new highlyskilled jobs. ${ }^{6}$

Supporting research initiatives in Australia are the four NHMRC government funding schemes, which were restructured in 2017 and implemented throughout 2018$19 .{ }^{11}$ The restructuring of the schemes was implemented due to a large volume of grant applications having low success rates. Therefore, to minimise the burden on researchers, the grant application process was streamlined so researchers could spend the majority of their time producing high-quality research. Despite the restructure, the changes to the Strategic and Leveraging Grants, which has a dedicated stream for clinical trials and cohort studies, was minimal, increasing the acceptance rate from $20 \%$ to $30 \%$. It should be noted that NHMRC government-funded clinical trials and cohort studies are required to meet their funding requirements, which place a high importance on quality procedures to achieve quality outcomes. As such, the large amount of Australian funding to support research relies largely on well-managed data to ensure Australian research is transparent and replicable.

\section{Unique Strengths, Challenges, and Regulations in Australia}

The National Statement on Ethical Conduct in Human Research (the National Statement) 2007 (updated 2018) ${ }^{12}$ is one of the main standards applicable to human research. The National Statement (sections 3.1.44-3.1.50) indicates that researchers should develop, as early as possible, a data management plan for all stages of the research data from generation through to re-use. The National Statement compliments the International Council for Harmonisation (ICH) Good Clinical Practice (GCP) guideline. ${ }^{13}$ However, a challenge for Australian data managers is inconsistency between the GCP guidelines and the National Statement. It is recommended to Australian researchers that if there is inconsistency between the National Statement and GCP guidelines, the National Statement should take precedence. Another challenge is that the two main government research bodies neither enforce nor monitor policy frameworks for data management, making it difficult to determine national compliance. ${ }^{14}$

Australia is unique for its open data environment (Australian National Data Service (ANDS)), which includes health and medical data. ${ }^{15}$ ANDS provides users with access to guides on how to publish and share sensitive data, and recommended health data repositories are utilised. Once sensitive human and personal data that are collected from clinical trials are de-identified, they can be legally shared under the Privacy Act $1988 .{ }^{16}$ Funding guidelines, such as the NHMRC Open Access Policy, ${ }^{17}$ the National Statement, ${ }^{12}$ the Australian Code for the Responsible Conduct of Research, 2018 (the Code), ${ }^{18}$ and the Australian Research Council (ARC) Open Access Policy ${ }^{19}$ encourage data deposition in a publicly accessible repository. Clinical data managers are critical throughout this process to assist with the data integration and system validation of data repositories. Publicly accessible data repositories rely on high-quality data and ideally low numbers of missing data to maximise the amount of data suitable for analysis and improved practices for the biomedical sciences. Therefore, Australia is at the forefront of creating a cohesive national collection of research resources and a data-rich environment that will enable new and more effective research.

The latest report by the Australian New Zealand Clinical Trial Registry (ANZCTR) has found that the number of registered intervention (clinical) trials and observational studies conducted in Australia was 12,329 per capita, ranking Australia 10th out of 25 countries over the last decade..$^{20}$ In the top 10 countries, only three (including Australia) were not part of Europe, and further, Australia ranked above the US, which is in 19th place. The ANZCTR report also stated that $33 \%$ of Australian registered clinical studies recruit participants from multiple countries. ${ }^{20}$ Australian clinical data managers are exposed to a range of cutting-edge clinical studies across a range of hospitals, government agencies, and not-for-profit organisations. However, researchers need to consider the data limitations associated with sharing data collected in another country and who legally owns the data.

\section{Recruitment, Training, and Education of Australian Clinical Data Management/Data Science Professionals}

The Code ${ }^{18}$ recognises good data management practice includes the ownership, storage, retention, and accessibility of data. According to the Code, it is the responsibility of the institutions to provide training, such as GCP. There are GCP training and courses available both online and in person (e.g., Association of Regulatory and Clinical Scientists (ARCS) Australia ${ }^{21}$ and PRAXIS Australia ${ }^{22}$ ). However, in Australia an emphasis needs to be placed on available training courses that cater to the clinical researchers' different levels of expertise as well as the different roles in CDM. It should be noted that knowledge and skills related to how to manage and monitor research data are not typically part of tertiary training in Australia.

Clinical data managers require a diverse skillset, including teamwork, written and oral communication, computer literacy, project management, database, and analytical skills. Due to their range of skills, data managers in the past have often had a multitude of tasks to complete and organisational responsibilities. It is only recently that CDM has evolved to have a stronger focus on data quality. 
In Australia, formal online training courses and/or face-toface educational workshops are warranted. CDM needs to be on par with other professional specialties (e.g., project managers, study managers, clinicians, and biostatisticians) with whom clinical data managers interact alongside daily. On the other hand, suitable options for training students are also needed and should incorporate mandatory research data management modules for higher degree research students to complete prior to commencing their research. Modules could be online and include topics on how to write a data management plan, how to organise and manage your data files, available data storage options, archiving your data, and organisational and government policies. Such offerings would enable students to develop basic project management, computational, and organisational skills before entering the workforce.

In a recent qualitative study, Australian clinical researchers expressed a lack of guidance, education, and training in relation to data management and monitoring. ${ }^{5}$ This study suggested that clinical data managers in both academic and industry settings have received either on-the-job training or self-paced learning focusing on specific work procedures. This doesn't necessarily mean that GCP training was not conducted. Commonly in Australia, clinical studies conduct in-house training related to standard operating procedures (SOPs) and the project-specific manual of procedures (MOPs), but these are rarely published. Without clear guidance on evidence-based practice or adequate training tailored to the Australian clinical context, researchers can only rely on their own judgements to manage data, which is a suboptimal operation. In an attempt to overcome some of these issues, a recent article ${ }^{23}$ has listed freely available and easy-to-use resources for the development and implementation of data management and monitoring practices for clinical studies in Australia.

\section{Conclusion}

In summary, CDM in Australia has progressed quickly due to the demands from the pharmaceutical industry. This is largely due to the drive to fast-track the development of pharmaceutical products, which continue to accelerate. In response, Australian regulatory agencies have put in place standardised guidance documents. This is to ensure that the implementation of quality systems will generate and manage high quality data outcomes. Positively impacting these processes and systems is the development of IT, which has improved the speed of collecting and the quality of data generated. However, one of the largest challenges that Australian data managers have faced is the planning for and implementation of CDM systems in a constantly changing operational environment as the rapid pace of IT developments outdate existing infrastructure. Therefore, CDM has had to strike a balance between the constraints of existing, and at times dated, systems and the expectations from newly developed systems. In parallel, many Australian CDM professionals have had limited formal training available to them whilst advocating for CDM to be acknowledged as a professional specialty in itself. It is clear that clinical data managers in Australia have and will continue to play a pivotal role in collecting, processing, and managing clinical data. Australian data managers will aid in the discovery of new treatments for diseases and will work towards improving population health.

\section{Funding Information}

The authors received no financial support for the research, authorship, and/or publication of this article.

\section{Competing Interests}

The authors have no competing interests to declare.

\section{References}

1. Houston L, Probst Y, Yu P, Martin A. Exploring Data Quality Management within Clinical Trials. Applied Clinical Informatics. 2018; 9(1): 72-81. DOI: https://doi.org/10.1055/s-0037-1621702

2. Houston L, Yu P, Martin A, Probst Y. Heterogeneity in clinical research data quality monitoring: A national survey. Journal of Biomedical Informatics. 2020; 108: 103491. DOI: https://doi.org/10.1016/j. jbi.2020.103491

3. Houston L, Martin A, Yu P, Probst Y. Timeconsuming and expensive data quality monitoring procedures persist in clinical trials: A national survey. Contemporary Clinical Trials. 2021; 103: 106290. DOI: https://doi.org/10.1016/j.cct.2021.106290

4. National Health and Medical research Council (NHMRC). Australian research Council (ARC) and Universities Australia (UA). Management of Data and Information in Research: A guide supporting the Australian Coide for the Responsibile Conduct of research. https://www.nhmrc.gov.au/sites/default/ files/documents/attachments/Management-ofData-and-Information-in-Research.pdf. Published 2019. Accessed 09 July 2021.

5. Houston L, Martin A, Yu P, Probst Y. Clinical researchers' lived experiences with data quality monitoring in clinical trials: A qualitative study. BMC Medical Research Methodology. 2021; 21(187). DOI: https://doi.org/10.1186/s12874-021-01385-9

6. MTP Connect and LEK Consulting. Clinical Trials in Australia: The economic profile and competitive advantage of the sector. https://www.mtpconnect. org.au/clinicaltrials. Published 2017. Accessed 03 March 2021.

7. Department of Health, Australian Government. Clinical Trials. https://www1.health.gov.au/ internet/main/publishing.nsf/Content/ClinicalTrials. Last updated 04 March 2021. Accessed 13 July 2013.

8. Thiers FA, Sinskey AJ, Berndt ER. Trends in the globalization of clinical trials. Nature Reviews Drug Discovery. 2008; 7(1): 13-14. DOI: https://doi. org/10.1038/nrd2441

9. Department of Health, Australian Government. Medical research. https://www.health.gov.au/ health-topics/medical-research. Accessed 13 July 2021. 
10. Collen MF. Clinical research databases-A historical review. Journal of medical systems. 1990; 14(6): 323344. DOI: https://doi.org/10.1007/BF00996713

11. Design and development of an evaluation framework for the NHMRC: Review of international practice, overview of NHMRC's data and grant management systems, and an evaluation framework and metrics. https://www.rand.org/ pubs/research_reports/RR2984.html. Published 2019. Accessed 04 March 2021.

12. National Health and Medical Research Council (NHMRC), the Australian Research Council and Universitites Australia. National Statement on Ethical Conduct in Human Resarch 2007 (Updated 2018). Commonwealth of Australia (2018). Accessed 2 September 2019.

13. International Council on Harmonisation (ICH) of technical requirements for registration of pharmaceuticals for human use 2016, E6(R2) Good Clinical Practice (GCP). https://database.ich. org/sites/default/files/E6_R2_Addendum.pdf. Accessed 30 June 2016.

14. Krahe MA, Toohey J, Wolski M, Scuffham PA, Reilly S. Research data management in practice: Results from a cross-sectional survey of health and medical researchers from an academic institution in Australia. Health Information Management. 2020; 49(2-3): 108-116. DOI: https://doi. org/10.1177/1833358319831318

15. Australian National Data Service (ANDS). Health and medical data. https://www.ands.org. au/working-with-data/sensitive-data/medical-andhealth. Accessed 05 March 2021.

16. Australian Government. Privacy Act 1988. https:// www.legislation.gov.au/Details/C2021C00024 Accessed 04 March 2021.
17. National Health and Medical Research Council (NHMRC). Open Access Policy (2018). https:// www.nhmrc.gov.au/about-us/resources/openaccess-policy. Published 2018. Accessed 05 March 2021.

18. National Health and Medical Research Council (NHMRC), Australian Research Council (ARC) and Universities Australia (UA). Australian Code for the Responsible Conduct of Research (2018). Accessed 04 March 2021.

19. Australian Research Council (ARC). Open Access Policy (2017). https://www.arc.gov.au/ policies-strategies/policy/arc-open-access-policyversion-20171. Published 2017. Accessed 05 March 2021.

20. Australian New Zealand Clinical Trials Registry (ANZCTR). The clinical trials landscape in Australia 2006-2015 (2017). https://www.anzctr. org.au/docs/ClinicalTrialsInAustralia2006-2015. pdf. Published 2017. Accessed 28 September 2019.

21. Association of Regulatory and Clinical Scientists (ARCS) Australia. Applied GCP Training for Investigational Sites and Sponsor Representatives E6(R2) Certificates 1,2 \& 3. https://www.arcs.com. $\mathrm{au} /$ events/category/online-learning. Accessed 18 November 2020.

22. PRAXIS Australia. Promoting Ethics and Education in Research. https://praxisaustralia.com.au/. Accessed 18 November 2020.

23. Houston L, Cortie CH, Probst Y, Meyer BJ. Improving data monitoring in Australian clinical trials and research: Free resources and templates. Clinical Trials. 2021; 17407745211026726. DOI: https://doi. org/10.1177/17407745211026726

How to cite this article: Houston L, Probst Y. Clinical Data Management: A Review of Current Practice in Australia. Journal of the Society for Clinical Data Management. 2021; 1(1): 15, pp. 1-5. DOl: https://doi.org/10.47912/jscdm.62

Copyright: ( $) 2021$ SCDM publishes JSCDM content in an open access manner under a Attribution-Non-Commercial-ShareAlike (CC BY-NC-SA) license. This license lets others remix, adapt, and build upon the work non-commercially, as long as they credit SCDM and the author and license their new creations under the identical terms. See https://creativecommons.org/licenses/by-nc-sa/4.0/. 